“ (C) 2017 IEEE. Personal use of this material is permitted. Permission from IEEE must be obtained for all other uses, in any current or future media, including

reprinting/republishing this material for advertising or promotional purposes, creating new collective works, for resale or redistribution to servers or lists, or reuse of any copyrighted component of this work in other works." 


\section{A Fuzzy Based Lagrangian Twin Parametric- Margin Support Vector Machine (FLTPMSVM)}

\author{
Deepak Gupta \\ Computer Science \& Engineering \\ NIT Arunachal Pradesh, \\ Yupia, India \\ deepakjnu85@gmail.com
}

\author{
Parashjyoti Borah \\ Computer Science \& Engineering \\ NIT Arunachal Pradesh, \\ Yupia, India \\ parashjyoti@hotmail.com
}

\author{
Mukesh Prasad \\ Centre of Artificial Intelligence \\ School of Software, FEIT \\ University of Technology Sydney, \\ Sydney, Australia \\ mukesh.nctu@gmail.com
}

\begin{abstract}
In the spirit of twin parametric-margin support vector machine (TPMSVM) and support vector machine based on fuzzy membership values (FSVM), a new method termed as fuzzy based Lagrangian twin parametric-margin support vector machine (FLTPMSVM) is proposed in this paper to reduce the effect of the outliers. In FLTPMSVM, we assign the weights to each data samples on the basis of fuzzy membership values to reduce the effect of outliers. Also, we consider the square of the 2norm of slack variables to make the objective function strongly convex and find the solution of the proposed FLTPMSVM by solving simple linearly convergent iterative schemes instead of solving a pair of quadratic programming problems as in case of SVM, TWSVM, FTSVM and TPMSVM. No need of external toolbox is required for FLTPMSVM. The numerical experiments are performed on artificial as well as well known real-world datasets which show that our proposed FLTPMSVM is having better generalization performance and less training cost in comparison to support vector machine, twin support vector machine, fuzzy twin support vector machine and twin parametric-margin support vector machine.
\end{abstract}

Keywords-Support vector machine; Twin support vector machine; Parametric-margin model; fuzzy membership; Iterative method

\section{INTRODUCTION}

One of the popular machine learning algorithms, support vector machine (SVM) [1] is an excellent kernel-based tool used in past few decades for wide variety of applications like text categorization [2], handwritten digit recognition [3], activity detection[4], stock exchange prediction [5], braincomputer interface [6], credit scoring [7] etc.

The computational complexity of SVM depends on solving a large sized quadratic programming problem (QPP) i.e. $\mathrm{O}\left(\mathrm{m}^{3}\right)$ where $m$ is the number of training data samples. This is the main disadvantage of this method for large scale datasets. Based on same principle, an efficient approach called twin support vector machine (TWSVM) has been proposed by Jayadeva et al. [8] to reduce the training cost and improve the generalization performance where it finds two non-parallel hyperplanes by solving two smaller sized QPPs instead of finding a single hyperplane by solving a single larger one in case of SVM, which results in a reduced training cost by approximately four times [8]. A least squares variant of SVM, called least squares support vector machine (LSSVM) [9], has been proposed to decrease the training cost. Mangasarian and
Musicant [10] has proposed an iterative method based on an implicit Lagrangian formulation and named it Lagrangian support vector machine (LSVM). Further, Balasundaram et al. [11] proposed a new approach for training Lagrangian twin support vector machine using unconstrained convex minimization. For Heteroscedastic noise structure, recently, Hao [12] has proposed a novel approach termed as parametricmargin $v$-support vector machine (Par- $v$-SVM) which is based on $v$-support vector machine ( $v$-SVM) [13]. Further, Peng [14] has proposed a novel approach, twin parametric-margin support vector machine (TPMSVM), where it solves two smaller sized QPPs instead of solving a single larger QPP as in case of Par- $v$-SVM. Hence, the training cost of TPMSVM is much lesser than Par-v-SVM.

In all the techniques discussed above, all the data samples belonging to one class contribute equally in finding the final classifier. But presence of outliers and noise in real-world datasets can effect in determining a more appropriate classifier. Hence, to lessen the effect of outliers and noise in finding the resultant classifier, a fuzzy-based SVM algorithm (FSVM) was proposed by Lin et al. [15]. In their algorithm, each training point is assigned a membership value which can be calculated using a suitable membership function depending on the nature of the problem. Samples with higher importance get a higher membership value whereas those who have less importance get a lower membership value. In due course, many variants based on FSVM have been proposed. Batuwita \& Palade [16] has proposed FSVMs for class imbalance learning (FSVM-CIL) to handle the problem of class imbalance. Furthermore, Tsujinishi et al. [17] has proposed a fuzzy least squares support vector machine for multiclass problems. Similarly, Wang et al. [18] has proposed a model Bilateral-weighted FSVM (B-FSVM). To solve bankruptcy prediction problem, a new fuzzy SVM is proposed [19]. Further, a fuzzy least squares support vector machine for object tracking is proposed by Zhang et al. [20].

In this paper, a new technique is proposed, termed as fuzzy based Lagrangian twin parametric-margin support vector machine (FLTPMSVM) to handle the outlier points which uses fuzzy membership values in decision learning. To find the resultant decision classifier, our proposed method FLTPMSVM solves simple linearly convergent iterative schemes instead of solving a pair of quadratic programming problems (QPPs) as in TWSVM, FTSVM and TPMSVM. Here, we are using MOSEK toolbox to solve the QPPs in 
SVM, TWSVM, FTSVM and TPMSVM. There is no need to use any external toolbox for our proposed FLTPMSVM. Hence, FLTPMSVM improves the generalization performance of the decision surface and takes less training time in comparison to others methods. Moreover, we have presented a comparative analysis of results in terms of classification accuracy and training time of SVM, TWSVM, FTSVM and TPMSVM with our method for synthetic and real-world datasets.

\section{RELATED WORK}

\section{A. Support Vector Machine (SVM)}

Let us suppose $\mathrm{X}$ is the input matrix of training samples and $Y$ is the label vector. Let us consider input matrices $\mathrm{X}_{1}$ and $\mathrm{X}_{2}$ of size $l_{1} \xi$ and $l \xi n, \quad l_{1}$ is the number of $n \quad$ where

data points belonging to positive class and $l_{2}$ denotes the

number of data points belonging to the negative class. such that the total number of data samples $m=l+l$ and $n$ is the total

$$
e_{1} \alpha_{2}
$$

number of attributes. The non-linear SVM maps the sample $x$ to a higher dimensional feature space using a mapping function $\chi \pi$ and finds the hyperplane $\chi \pi(x) w+b=0$ by (.)

solving the following formulation

$$
\min _{\zeta}^{1}\|w\|^{2}+C e^{t}
$$

2

subject to $Y(\chi \pi(X) w+e b)+\zeta \quad e$,

$$
\zeta 0
$$

where $\zeta$ represents slack variables; $C$ is the penalty parameters; $e$ is a unit vector of suitable dimension.

After finding the Lagrangian formulation of equation (1) and applying the Karush-Kuhn-Tucker (K.K.T) necessary and sufficient conditions [21], the Wolfe dual of (1) is derived as

$$
\begin{array}{cl}
\max e^{t} \alpha & { }^{1} \alpha^{t} Y X X^{t} Y \alpha \\
& 2 \\
\text { subject to } & \quad \boldsymbol{\alpha}^{t} \boldsymbol{Y} \boldsymbol{\alpha}=0,0 \\
& \alpha \quad C
\end{array}
$$

subject to $\left(K\left(\mathrm{X}, D^{\mathrm{t}}\right) w+e b\right)+e, \quad 0$

where $\}$, represent slack $\quad C_{1}, C_{2}$ are penalty variables;

parameters; $D=[\mathrm{X} ; \mathrm{X}] ; e, e$ are vectors of suitable dimension having ${ }^{1}{ }^{2}{ }^{2}{ }^{1}{ }^{2}$ values as 1 's and $K x^{t} D^{t}=k x x \quad k x x$ is a row vector in $R^{m}$, where $(, \quad) \quad\left(\left(,_{1}\right), \ldots,\left(,,_{m}\right)\right)$

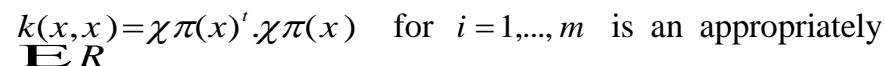
chosen kernel.

By introducing the Lagrangian functions of problems (4) \& (5) and applying the Karush-Kuhn-Tucker (K.K.T) necessary and sufficient conditions [21], the Wolfe dual of (4) and (5) are written as

$$
\begin{array}{cccc}
\max & e^{t} \alpha & 1 & \alpha^{t} H\left(G^{t} G\right)^{1} H^{t} \alpha \\
& - & \\
2 & & 2 & 1
\end{array}
$$

$$
\begin{aligned}
& \text { subject to } \quad \begin{array}{llll}
0 & \alpha_{1} & C_{1}
\end{array} \\
& \max { }^{t} \underline{1} t t_{t} \\
& \propto^{2} G(H H) G
\end{aligned}
$$

subject to $\quad 0 \quad \alpha \quad C$

$$
\begin{aligned}
& \text { where } \quad G=\left[K\left(\mathrm{X}_{1}, D\right) e_{1}\right], \quad H=\left[K\left(\mathrm{X}_{2}, D\right) e_{2}\right] \text {; }
\end{aligned}
$$

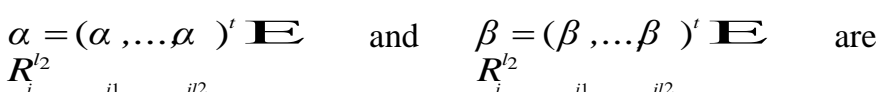

Lagrangian multipliers for $i=1,2$.

We compute the values of $w_{1} w_{2}, b_{1} \quad b_{2}$ using the following equations as and

$\mathrm{Tw}$

$$
\begin{aligned}
& { }^{1}=\left(G^{t} G+\delta I\right)^{-1} H^{t} \alpha^{1} \\
& \Lambda^{b_{1} \vartheta} \\
& \mathrm{T} w_{2}{ }^{t}{ }^{-1}{ }^{t} \\
& =(H H+\delta I) \quad G \alpha_{2} \\
& \Lambda^{b_{2}} \vartheta
\end{aligned}
$$

where $\boldsymbol{O}$ is a small positive integer value and $I$ is an identity 
where $\boldsymbol{\alpha}$ is the vector of Lagrangian multipliers. A new $\mathbf{E} R^{m}$

data point $x \mathbf{E} R^{n}$ is assigned to a given class ' $i$ ' as follows

$$
\begin{aligned}
& \text { class } i=\operatorname{sign} \mid \chi \pi(x)^{t} w+ \\
& b \mid
\end{aligned}
$$

\section{B. Twin Support Vector Machine (TWSVM)}

In TWSVM [8], two non-parallel hyperplanes are obtained such that each of them is nearer to one of the classes and as far as possible from the other class. In non-linear case, twin support vector machine finds a pair of non-parallel hyperplanes $K(x, D) w_{1}+b_{1}=0 \quad$ and $\quad K(x, D) w_{2}+b_{2}=0$ from the solution of the following QPPs

$$
\begin{aligned}
& \min _{2}^{1}\left\|K\left(\mathrm{X}, D^{t}\right) w+e b\right\|^{2}+C e^{t} \\
& \text { subject to } \left.\left(K\left(\mathrm{X}_{2}, D^{t}\right) w_{1}+e_{2} b_{1}\right)+\zeta \quad, \quad\right\} \\
& \min _{2}^{1}\left\|K\left(\mathrm{X}, D^{t}\right) w+e b\right\|^{2}+C e^{t} \\
& \frac{2}{2} \quad{ }_{2} \quad{ }_{2}
\end{aligned}
$$

matrix of appropriate dimension [8].

Further, a test data point $x \mathbf{E}$ is assigned to a given $R^{n}$

class ' $i$ ' by using the following formula

$$
\text { class } i=\min \left|K\left(x_{t}, D_{t}\right) w_{i}+b_{i}\right| \quad \text { for } i=1,2 \text {. }
$$

\section{Fuzzy Twin Support Vector Machine (FTSVM)}

In FTSVM, weights are given to the different data samples on the basis of fuzzy membership values and the training gets biased towards the samples of interest. To calculate the fuzzy membership, we have considered the centroid measure for the data samples of each class where the membership values are assigned based on the distance of the data points from the centroid of that class [22]. The membership values are used as a basis for giving weights to the error tolerance parameter $C$

for every data point.

The fuzzy membership function for centroid based membership is written as 


$$
\text { mem=1 } \frac{d_{c e n}}{\max \left(d_{c e n}\right)+}
$$

where $d_{c e n}$ is the Euclidean distance of each data point from the centroid of its class i.e. $d_{c e n}=\left\|x_{+} \quad x_{i}\right\|$ if $\quad y_{i}=1$ and otherwise $d_{c e n}=\left\|x \quad x_{i}\right\|$, where $x_{+}$and $x$ are the centroids

of positive and negative class respectively, and $\boldsymbol{O}$ is a small positive integer value.

In FTSVM, the non-linear hyperplanes are obtained through the following problems

$$
\begin{aligned}
& \min { }^{1}\left\|K\left(\mathrm{X}, \mathrm{D}^{\mathrm{t}}\right) w+e b\right\|^{2}+C S^{t} \xi \\
& 2 \\
& 0 \text { subject to }\left(K\left(\mathrm{X}_{2}, \mathrm{D}\right) w_{1}+e_{2} b_{1}\right)+\xi \quad e_{2}, \zeta
\end{aligned}
$$

$$
\begin{aligned}
& \min { }^{1}\left\|K\left(\mathrm{X}, \mathrm{D}^{\mathrm{t}}\right) w+e b\right\|^{2}+C S^{t} \eta \\
& 2
\end{aligned}
$$

subject to $\left(K\left(\mathrm{X}_{1}, \mathrm{D}\right) w_{2}+e_{1} b_{2}\right)+\eta \quad e_{1}, \quad 0$

where $S, S$ are vectors having the membership values of the

data samples of the positive and the negative class respectively.

By introducing the Lagrangian functions of problems (12) \& (13) and applying the Karush-Kuhn-Tucker (K.K.T) necessary and sufficient conditions [21], the Wolfe dual of

(12) and (13) are written as

$$
\begin{aligned}
& 1 \\
& \min \frac{-}{2} \alpha_{1} H(G G) \quad H \alpha_{1} \quad e_{2} \alpha_{1}
\end{aligned}
$$$$
\text { subject to } 0 \quad \alpha_{1} \quad S_{1} C_{1}
$$

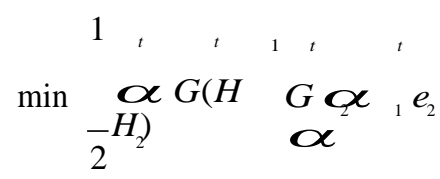

subject to $0 \quad \alpha_{2} \quad S_{2} C_{2}$

$$
\left.\min { }^{1} w^{t} w+\varpi_{1} e^{t}(\chi \pi(\mathrm{X}) w+e b)+{ }^{C_{1}} e^{t}\right\}
$$

$$
\begin{array}{llllllllll}
\overline{2} & 1 & 1 & \bar{l}_{2} & 2 & 2 & 1 & 2 & 1 & \bar{l}_{1}
\end{array}
$$

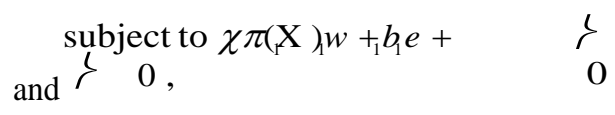

$$
\text { and }
$$

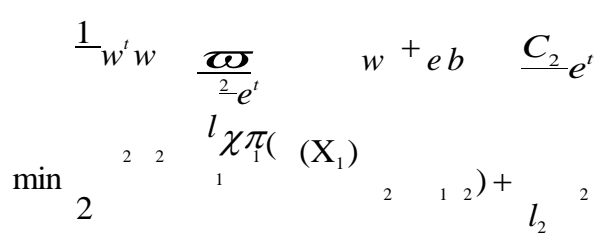

$$
\begin{aligned}
& \text { subject to } \chi \pi\left(\mathrm{X}_{2}\right) w_{2}+b_{2} e_{2} \quad 0 \text {. } \\
& 0
\end{aligned}
$$

One can write (17) and (18) in the form of dual QPPs by considering the Lagrangian multipliers and apply the KKT necessary and sufficient conditions, which are

$$
\begin{aligned}
& \min ^{1} \propto{ }^{1} \propto-{ }^{1} e^{t} K{ }^{\mathrm{t}} \propto \\
& \overline{2} \overline{1}^{1}\left(\mathrm{X}_{1}, \mathrm{X}_{1}\right)_{1} l_{2} l_{2}\left(\mathrm{X}_{2}, \mathrm{X}_{1}\right)_{1}
\end{aligned}
$$

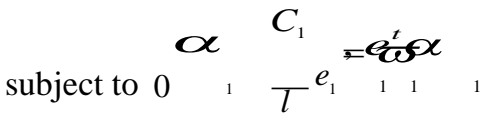

and

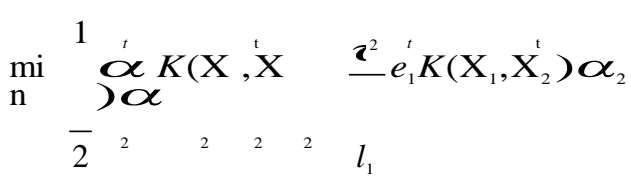

$$
\begin{aligned}
& \underset{\alpha}{\text { subject to } 0 \quad 2 \quad \frac{C_{2}}{l}=\underset{\varpi}{t} \boldsymbol{\alpha}}
\end{aligned}
$$

respectively. After computing the values of $\boldsymbol{\chi}_{2}$ by solving , $\alpha$

the QPPs (19) \& (20), one can find the solution of $(w, b)$ and $\left(w_{2}, b_{2}\right)$ in the following manner

$$
\begin{aligned}
& w_{1}=\chi \pi\left(\mathrm{X}_{1}\right) \alpha_{1}^{\mathbf{1}_{1}} \underset{1}{\chi \pi(\mathrm{X})^{t} e} \text { and }_{1} b=\quad \Lambda \chi \pi\left(\mathrm{X}_{1}\right) w_{1} \\
& \overline{l_{2}} \quad \overline{\left|N_{1}\right|}{ }^{i \mathrm{E}|N 1|}
\end{aligned}
$$

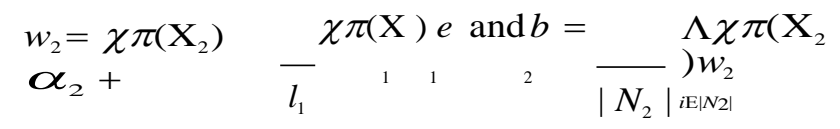

subject to 0 
Further, we compute the values of $w_{1}, w_{2}, b_{1}$ and $b_{2}$ as

$\mathrm{T} w$

$\mathrm{Tw}$

${ }^{1}=\left(G^{t} G+\delta I\right)^{-1} H^{t} \alpha$ and ${ }^{2}=\left(H^{t} H+\delta I\right)^{-1} G^{t} \alpha$

$k_{1}$ $k_{2}$
9

$\vartheta$

The resultant classifier is obtained by using the equation (10).

D. Twin parametric-margin support vector machine

\section{(TPMSVM)}

Recently, Peng [14] has proposed an efficient twin parametric-margin support vector machine which is an efficient learning approach of par- $v$-SVM. It finds two hyperplanes $\quad f_{1}(x)=w_{1}^{t} \chi \pi(x)+b_{1} \quad f_{f_{2}}(x)=w_{2} \chi \pi(x) \quad$ in the feature space which are obtained by the following formulations: where

$N_{i}$ is the index set of samples satisfying

I $\quad l_{i}$

for $i=1,2$. Finally, for any input sample $x \mathbf{E} R^{n}$, the classifier

is given by

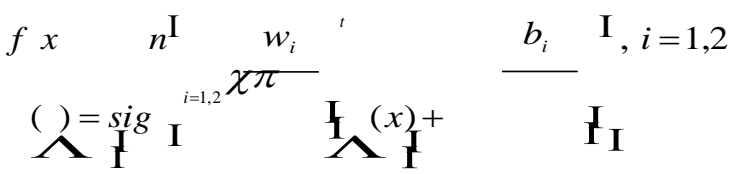

$$
\begin{aligned}
& \left\|w_{i}\right\| \quad \quad \quad\left\|w_{i}\right\|
\end{aligned}
$$

\section{PROPOSED FUZZY BASED LAGRANGIAN TWIN PARAMETRIC-MARGIN SUPPORT VECTOR MACHINE (FLTPMSVM)}

In this section, motivated by the work of Peng [14], we propose a new variant of TPMSVM which is based on TWSVM, called fuzzy based Lagrangian twin parametricmargin support vector machine where fuzzy membership values are calculated as similar in FTSVM. For the non-linear 
case, our proposed FLTPMSVM finds the positive and I

negative parametric margin hyperplanes $K(x, D) w_{1}+b_{1}=0$ and $K(x, D) w_{2}+b_{2}=0$.

In order to find the both parametric-margin hyperplanes, we have considered the following formulation

$\min _{\zeta{ }^{t} \zeta}^{1}\left(w^{t} w+b^{2}\right)+\varpi e^{t}\left(K\left(\mathrm{X}, D^{t}\right) w+e b\right)+{ }^{S_{1} C_{1}}$ $\begin{array}{llllllllllll}2 & 1 & 1 & 1 & 1 & 2 & 2 & 1 & 2 & 1 & 2\end{array}$

subject to $\left(K\left(\mathrm{X}_{1}, D\right) w_{1}+e_{1} b_{1}\right)+\zeta 0$, and

$$
\begin{aligned}
& \min { }_{-}^{1}\left(w^{t} w+b_{t}^{2}\right) \varpi e^{t}\left(K\left(\mathrm{X}, D^{t}\right) w+e b\right)+{ }_{2}^{S_{2} C_{2}} \\
& \begin{array}{llllllllllll}
2 & 2 & 2 & 2 & 2 & 1 & 1 & 1 & 1 & 2 & 2
\end{array} \\
& \text { subject to }\left(K\left(\mathrm{X}_{2}, D\right) w_{2}+e_{2} b_{2}\right) \quad 0 \text {. }
\end{aligned}
$$

The Lagrangian functions of (22) and (23) are given as

$$
\left.\underset{H u}{L}={ }^{1} u^{t} u+\varpi e^{t}+{ }_{(G u}^{C_{1}} S \zeta^{t} \xi \alpha^{t}+\zeta\right)
$$

$$
\begin{array}{llllllll}
1 & \overline{2} & 1 & 1 & 1 & 2 & 1 & \overline{2}
\end{array}
$$

and

$$
\begin{aligned}
& \left.L={ }_{-}^{1} u^{t} u \underset{G u}{\varpi} e^{t}+{ }_{(\mathrm{Hu}}^{C_{2}} S^{t} \boldsymbol{\alpha}^{t}\right) \\
& \begin{array}{lllllllllll}
2 & 2 & 2 & 2 & 2 & 1 & 2 & 2 & 2 & 2 & 2
\end{array} \\
& \mathrm{Tw} \quad \mathrm{T} w
\end{aligned}
$$$$
\text { where } u_{1}=u_{2}=2 \text {. }
$$

$$
\Lambda_{1} \vartheta \quad \Lambda b_{2} \vartheta
$$

Now, one can apply the Karush-Kuhn-Tucker (K.K.T) necessary and sufficient conditions to find the Wolfe dual of equations (22) and (23) as

$$
\begin{aligned}
& \min { }^{1} \alpha^{t} \mathrm{I} \underline{I}+G_{\alpha} G^{t} \quad H G \alpha e^{t}
\end{aligned}
$$

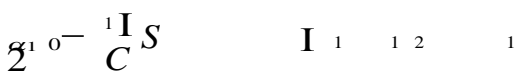

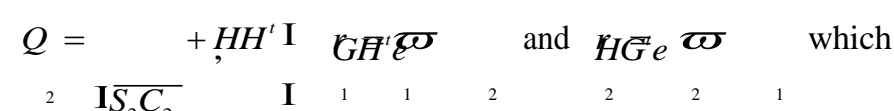

will lead to following pair of classical complementary problems [23]:

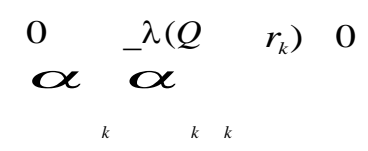

To find the solution of problems (29), we consider the

equivalent pair of problems: for any ${ }_{k}>0$, for $k=1,2$, the relations

$$
\begin{aligned}
& \left(Q_{k} \alpha_{k} r_{k}\right)=\left(Q_{k} \alpha_{k}\right. \\
& \left.{ }_{k} \boldsymbol{\alpha}_{k} \quad r_{k}\right)_{+}
\end{aligned}
$$

So, we can solve the problem (30) by writing as the following iterative scheme: for $i=0,1,2 \ldots$

$$
\begin{aligned}
& { }_{k}=Q_{k}\left(r_{k}+\left(Q_{k} \alpha_{k}{ }_{k} \boldsymbol{\alpha}_{k} r_{k}\right)_{+}\right) \\
& \text {i.e. }{ }^{i+1}{ }^{1}
\end{aligned}
$$

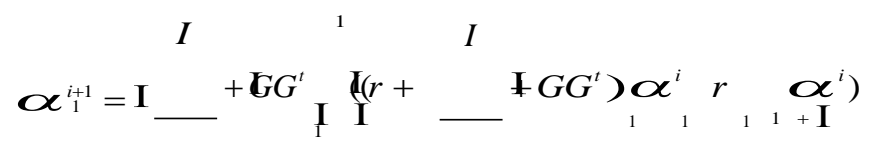

$$
\begin{aligned}
& S_{1} C_{1} \quad S_{1} C_{1}
\end{aligned}
$$

and

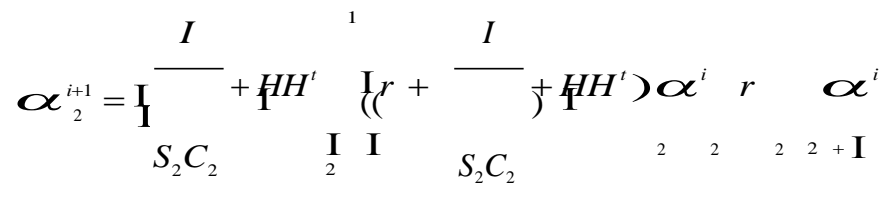

when $0<{ }_{1}<\frac{2}{C_{1}}$ and $0<{ }_{2}<\frac{2}{C_{2}}$, the above iterative schemes willconverge to the solutions $\boldsymbol{\alpha}$ and respectively $\alpha$

[24]. The final classifier is defined by the equation (21). 


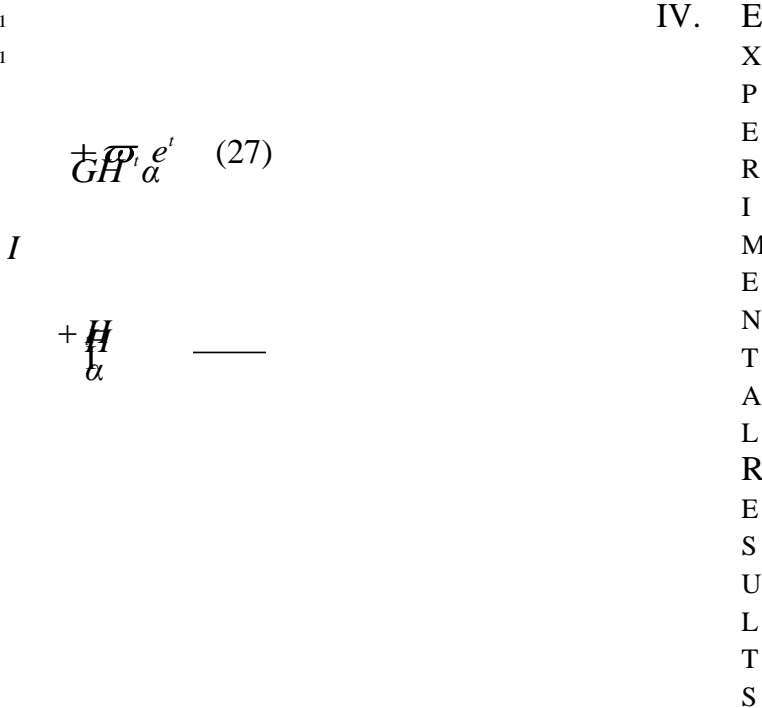

To

validate

the

effective

ness of

our

method,

we have

conducte

d an

experime

nt and

have

compare

d our

proposed

FLTPMS

VM with

SVM,

TWSVM

, FTSVM

and

TPMSV

$2^{2}{ }^{-}{ }^{2} \mathbf{I} S$

$\mathrm{M}$

I $\begin{array}{llll}2 & 2 & 1 & 2\end{array}$

After computing the values of $\propto \propto \alpha$ from (26) and and

(27), we find the positive and negative class hyperplanes $\left[K\left(\mathrm{X}_{1}, D^{t}\right) e_{1}\right] u_{1}=0$ and $\left[K\left(\mathrm{X}^{2}, D^{t}\right) e_{2}\right] u_{2}=0 \quad$ respectively ware $G u_{u_{1}}=G \boldsymbol{\alpha}_{1} \quad \varpi_{1} \boldsymbol{H} e_{2}$ and $u_{2}=\boldsymbol{H} \boldsymbol{\alpha}_{2}$

To predict the class of new data sample $x \mathbf{E}$ in case of $R^{n}$

FLTPMSVM, we find the class label by using equation (21).

These dual QPPs (26) and (27) are of the form

$$
\min ^{1} \alpha^{t} Q \alpha+r^{t} \alpha
$$

on well-known real-world datasets as well as one artificial dataset i.e. Ripley's dataset $[25]$. All the experiments are $3770 \mathrm{CPU}$ and 4 GB RAM, running Windows 7 operating system. The software package used is MATLAB R2008a along with MOSEK optimization toolbox for TWSVM, FTSVM and TPMSVM, available at https://www.mosek.com.

The datasets are normalized to the range $[0,1]$ before experiment is performed on them. In this experiment, we implemented all the methods for non-linear case using Gaussian kernel which is given by

$$
\begin{gathered}
K\left(x_{i}, x_{j}\right)=\exp \mathbf{I} \frac{\left\|x_{i} x_{j}\right\|^{2}}{\mathbf{I}} . \\
2 \alpha^{2}
\end{gathered}
$$




$$
Q_{1}=\mathrm{I} \frac{I}{\longrightarrow}+G G^{t} \mathbf{I}
$$

for $k=1,2$ respectively, where $\quad S_{1} C_{1}$ obtained

from the set $\left\{2^{5}, \ldots, 2^{5}\right\}, C \quad(i=1,2)$ are also obtained from

the set $\left\{10^{5}, \ldots, 10^{5}\right\}$ and for TPMSVM and FLTPMSVM, the 
optimum values of $\underset{C}{\boldsymbol{U}}(i=1,2)$ are selected from

$\{0.1, \ldots, 0.9\}$ by using 10 -fold cross-validation of the training data.

We set average accuracy and average training time as the performance evaluation criteria for all the algorithms. Statistical result analysis is performed on testing data to calculate the average accuracy, standard deviation of result and average training time. We have considered the artificiallygenerated Ripley's synthetic dataset. The Ripley's dataset is a synthetic dataset in $R^{2}$ that contains 250 training samples and

1000 samples for testing. In the figure, the positive class samples and the negative class samples are depicted using ‘ $\xi$ and '+' symbols respectively. Support vectors are marked using circles around them. The learning results of our experiment on artificial Ripley's dataset for SVM, TWSVM, FTSVM, TPMSVM and FLTPMSVM are shown in Figures 1(a-e).

One can observe that FLTPMSVM obtains better decision classifiers in comparison to SVM, TWSVM, FTSVM, and TPMSVM. In Table 1, we show the predicted accuracies, optimum parameters with learning time for FLTPMSVM with others methods. One can notice from Table 1 that our proposed FLTPMSVM has best classification result among these algorithms as well as the learning time of our proposed method is less which show that FLTPMSVM takes less

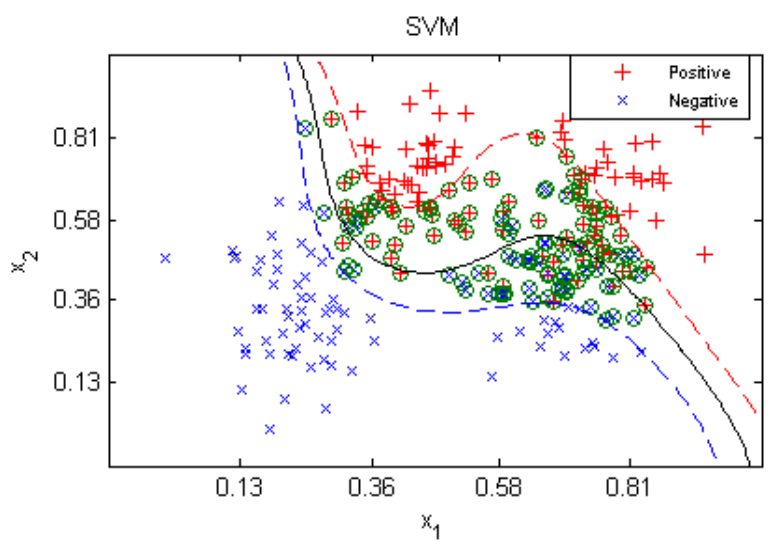

(a)

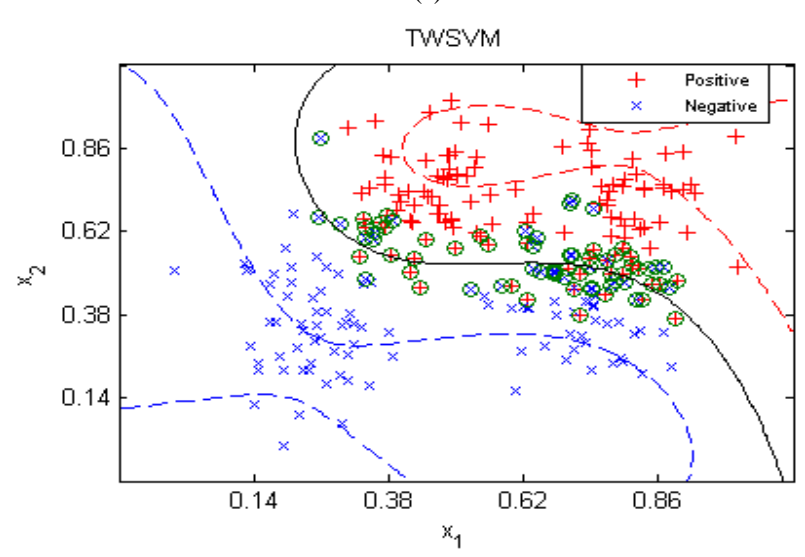

(b)

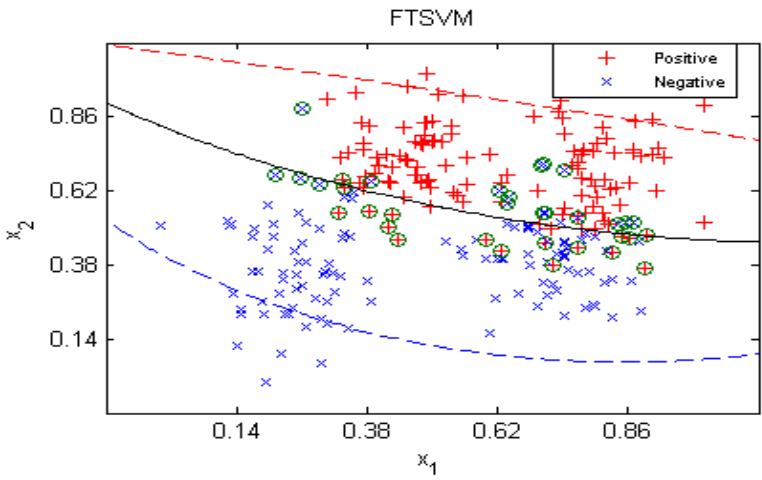

(c)

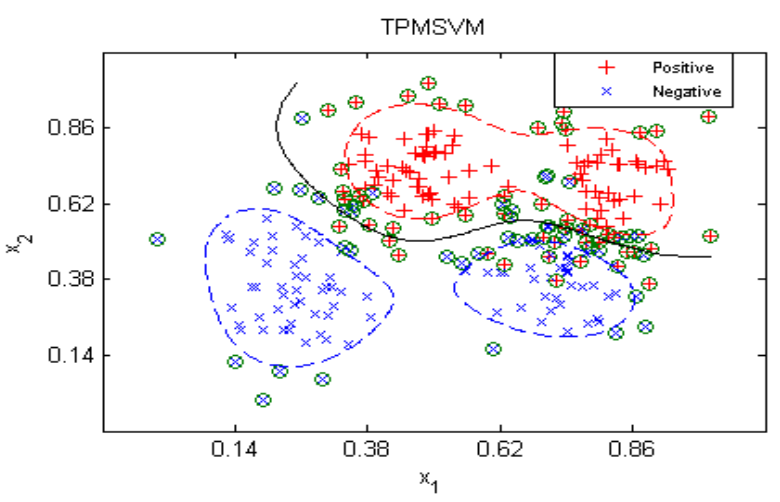

(d)

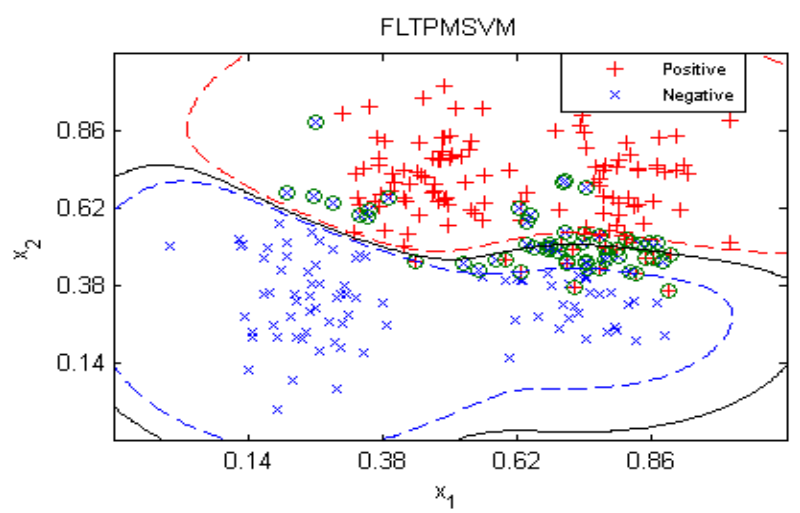

(e)

Fig. 1. Discriminant boundaries of FLTPMSVM with TWSVM, FTSVM and TPMSVM on Ripley's dataset using Gaussian kernel

computation time when compared with the other considered methods.

Further, we have considered 10 UCI benchmark wellknown real-world datasets i.e. Australian-Credit, BreastCancer, BUPA liver, Cleveland, Haberman, Heart-Statlog, Ionosphare, Pima-indians-diabetes, Transfusion, Wpbc from UCI repository [26]. The classification accuracy along with optimum parameters and training time of all algorithms are presented in Table 2. Our proposed FLTPMSVM is performed better in 6 out of 10 datasets. The solution of the proposed 
TABLE I. The result of FLTPMSVM, TWSVM, FTSVM and TPMSVM on Ripley’s dataset

\begin{tabular}{cccccc}
\hline Dataset & SVM & TWSVM & FTSVM & TPMSVM & \multicolumn{2}{c}{ FLTPMSVM } \\
(Train size, & $(C, \mu)$ & $\left(C_{1}=C_{2}, \mu\right)$ & $\left(C_{1}=C_{2}, \mu\right)$ & $(C=C=C, \mu, \varpi /$ & $\left(C=C_{1}=C_{2} \mu, \varpi / C\right)$ \\
Test size $)$ & Time & Time & Time & $C)_{2}$ & Time \\
\hline Ripley & 90.3 & 88.9 & 88.7 & Time & $\mathbf{9 0 . 9}$ \\
$(250 \times 2$, & $\left(10^{\wedge}-5,2^{\wedge} 2\right)$ & $\left(10^{\wedge} 0,2^{\wedge}-1\right)$ & $\left(10^{\wedge} 1,2^{\wedge} 1\right)$ & 90 & $\left(10^{\wedge} 0,2^{\wedge}-3,0.9\right)$ \\
$1000 \times 2)$ & 1.5464 & 0.124 & 0.1268 & $\left(10^{\wedge}-3,2^{\wedge}-3,0.4\right)$ & 0.1137 \\
& & & 0.1212 & \\
\hline
\end{tabular}

TABLE II. The results of FLTPMSVM with TWSVM, FTSVM and TPMSVM using Gaussian kernel on real-world datasets

\begin{tabular}{|c|c|c|c|c|c|}
\hline $\begin{array}{c}\text { Dataset } \\
\text { (Train size, Test } \\
\text { size) } \\
\end{array}$ & $\begin{array}{c}\text { SVM } \\
(C, \mu) \\
\text { Time } \\
\end{array}$ & $\begin{array}{c}\text { TWSVM } \\
\left(C_{1}=C_{2}, \mu\right) \\
\text { Time }\end{array}$ & $\begin{array}{c}\text { FTSVM } \\
\left(C_{1}=C_{2}, \mu\right) \\
\text { Time }\end{array}$ & $\begin{array}{c}\text { TPMSVM } \\
(C=C=C, \mu, \varpi / \\
C)_{2}\end{array}$ & $\begin{array}{c}\text { FLTPMSVM } \\
\left(C=C_{1}=C_{2} \mu, \varpi / C\right) \\
\text { Time }\end{array}$ \\
\hline & & & & Time & \\
\hline \multirow{3}{*}{$\begin{array}{l}\text { Australian-Credit } \\
(413 \times 14,277 \times 14)\end{array}$} & $79.4974 \pm 8.1208$ & $84.4444 \pm 8.5695$ & $\mathbf{8 5 . 2 1 1 6} \pm 7.811$ & $84.828 \pm 9.5534$ & $84.8413 \pm 5.8409$ \\
\hline & $\left(10^{\wedge}-5,2^{\wedge}-3\right)$ & $\left(10^{\wedge}-2,2^{\wedge} 1\right)$ & $\left(10^{\wedge}-5,2^{\wedge}-1\right)$ & $\left(10^{\wedge}-2,2^{\wedge} 3,0.1\right)$ & $\left(10^{\wedge}-3,2^{\wedge} 3,0.1\right)$ \\
\hline & 1.3881 & 0.1193 & 0.1435 & 0.1205 & 0.1176 \\
\hline \multirow{2}{*}{$\begin{array}{c}\text { Breast-Cancer } \\
(149 \times 9,550 \times 9)\end{array}$} & $96.3636 \pm 2.2677$ & $96.3636 \pm 3.3195$ & $96.7273 \pm 3.1840$ & $97.0909 \pm 2.5997$ & $97.2727 \pm 2.6068$ \\
\hline & $\begin{array}{c}\left(10^{\wedge}-5,2^{\wedge}-1\right) \\
5.4858\end{array}$ & $\begin{array}{c}\left(10^{\wedge}-5,2^{\wedge}-1\right) \\
04643\end{array}$ & $\begin{array}{c}\left(10^{\wedge}-5,2^{\wedge}-1\right) \\
04654\end{array}$ & $\left(10^{\wedge}-2,2^{\wedge} 0,0.2\right)$ & $\left(10^{\wedge}-2,2^{\wedge} 3,0.1\right)$ \\
\hline \multirow{3}{*}{$\begin{array}{c}\text { BUPA liver } \\
(241 \times 6,104 \times 6)\end{array}$} & $\begin{array}{c}\text { J.40J0 } \\
49.7273 \pm 18.388\end{array}$ & $\begin{array}{c}0.4643 \\
56.1818+18.615\end{array}$ & $\begin{array}{c}0.4654 \\
58.3636+207384\end{array}$ & $\begin{array}{r}0.4225 \\
\end{array}$ & 0.4131 \\
\hline & $\left(10^{\wedge}-5,2^{\wedge} 3\right)$ & $\begin{array}{c}56.1818 \pm 18.615 \\
\left(10^{\wedge}-2,2^{\wedge}-1\right)\end{array}$ & $\begin{array}{c}58.3636 \pm 20.7384 \\
\left(10^{\wedge} 0,2^{\wedge}-1\right)\end{array}$ & $\begin{array}{c}59.1818 \pm 18.1285 \\
\left(10^{\wedge} 0,2^{\wedge} 0,0.4\right)\end{array}$ & $\begin{array}{c}60.2727 \pm 27.289 / \\
\left(10^{\wedge}-4,2^{\wedge} 4,0.1\right)\end{array}$ \\
\hline & 0.2019 & 0.0248 & 0.0257 & 0.0222 & 0.0176 \\
\hline \multirow{2}{*}{$\begin{array}{c}\text { Cleveland } \\
(178 \times 13,119 \times 13)\end{array}$} & $78.1061 \pm 9.08$ & $83.1818 \pm 5.5762$ & $84.0909 \pm 6.0501$ & $79.0152 \pm 4.2564$ & $79.9242 \pm 7.9315$ \\
\hline & $\begin{array}{c}\left(10^{\wedge}-5,2^{\wedge}-2\right) \\
02595\end{array}$ & $\left(10^{\wedge} 0,2^{\wedge} 3\right)$ & $\begin{array}{c}\left(10^{\wedge}-5,2^{\wedge} 2\right) \\
00269\end{array}$ & $\left(10^{\wedge}-1,2^{\wedge} 5,0.2\right)$ & $\left(10^{\wedge} 0,2^{\wedge} 0,0.2\right)$ \\
\hline \multirow{3}{*}{$\begin{array}{c}\text { Haberman } \\
(183 \times 3,123 \times 3)\end{array}$} & $68.3974 \pm 12.4044$ & $75.7051 \pm 12.6211$ & $\begin{array}{c}0.0269 \\
74.8077 \pm 11.3109\end{array}$ & $\begin{array}{c}0.0259 \\
69.1667 \pm 12.8754\end{array}$ & $\begin{array}{c}0.0230 \\
\mathbf{7 5 . 8 3 3 3}+11.8779\end{array}$ \\
\hline & $\left(10^{\wedge}-5,2^{\wedge} 3\right)$ & $\left(10^{\wedge}-1,2^{\wedge} 0\right)$ & $\left(10^{\wedge}-5,2^{\wedge} 0\right)$ & $\left(10^{\wedge}-3,2^{\wedge}-5,0.5\right)$ & $\left(10^{\wedge}-2,2^{\wedge}-2,0.2\right)$ \\
\hline & 0.2761 & 0.0262 & 0.0328 & 0.0256 & 0.0241 \\
\hline \multirow{3}{*}{$\begin{array}{c}\text { Heart-Statlog } \\
(161 \times 13,109 \times 13)\end{array}$} & $79.8182 \pm 10.3012$ & $79.7273 \pm 12.182$ & $79.7273 \pm 12.9139$ & $81.5455 \pm 7.8326$ & $\mathbf{8 4 . 4 5 4 5} \pm 7.4035$ \\
\hline & $\left(10^{\wedge}-5,2^{\wedge} 0\right)$ & $\left(10^{\wedge}-2,2^{\wedge} 0\right)$ & $\left(10^{\wedge} 1,2^{\wedge} 2\right) 0.0297$ & $\left(10^{\wedge}-3,2^{\wedge} 0,0.5\right)$ & $\left(10^{\wedge}-2,2^{\wedge} 1,0.2\right)$ \\
\hline & 0.2209 & 0.0224 & $\mathbf{9 3 . 2 7 2 7} \pm 4.6592$ & 0.0226 & 0.0198 \\
\hline \multirow{3}{*}{$\begin{array}{c}\text { Ionosphare } \\
(246 \times 34,105 \times 34)\end{array}$} & $83.6364 \pm 11.5311$ & $\mathbf{9 3 . 2 7 2 7} \pm 4.6592$ & $\left(10^{\wedge}-2,2^{\wedge} 0\right)$ & $92.5455 \pm 7.2322$ & $\mathbf{9 3 . 2 7 2 7} \pm 7.8928$ \\
\hline & $\left(10^{\wedge}-5,2^{\wedge}-1\right)$ & $\left(10^{\wedge}-2,2^{\wedge} 0\right)$ & 0.028 & $\left(10^{\wedge}-2,2^{\wedge} 0,0.2\right)$ & $\left(10^{\wedge}-4,2^{\wedge} 0,0.1\right)$ \\
\hline & 0.2089 & 0.0287 & $77.6735 \pm 6.7968$ & 0.0231 & 0.0182 \\
\hline \multirow{3}{*}{$\begin{array}{l}\text { Pima-indians- } \\
\text { diabetes } \\
(307 \times 8,461 \times 8)\end{array}$} & $77.6781 \pm 8.1724$ & $77.8908 \pm 7.5103$ & $\left(10^{\wedge} 0,2^{\wedge} 2\right)$ & $75.7216 \pm 8.0453$ & $72.0352 \pm 9.2246$ \\
\hline & $\left(10^{\wedge}-5,2^{\wedge} 5\right)$ & $\left(10^{\wedge} 0,2^{\wedge} 1\right)$ & 0.3304 & $\left(10^{\wedge} 0,2^{\wedge} 2,0.2\right)$ & $\left(10^{\wedge}-2,2^{\wedge} 0,0.1\right)$ \\
\hline & 3.8896 & 0.3198 & $77.6667 \pm 20.3093$ & 0.2965 & 0.2780 \\
\hline \multirow{3}{*}{$\begin{array}{c}\text { Transfusion } \\
(448 \times 4,300 \times 4)\end{array}$} & $\mathbf{8 0 . 3 3 3 3} \pm 19.0807$ & $77.6667 \pm 19.5031$ & $\left(10^{\wedge}-5,2^{\wedge} 0\right)$ & $66 \pm 28.8376$ & $80 \pm 18.8562$ \\
\hline & $\left(10^{\wedge}-5,2^{\wedge} 4\right)$ & $\left(10^{\wedge}-1,2^{\wedge} 1\right)$ & 0.1357 & $\left(10^{\wedge}-2,2^{\wedge}-1,0.1\right)$ & $\left(10^{\wedge}-5,2^{\wedge} 1,0.5\right)$ \\
\hline & 1.6251 & 0.135 & $79.4643 \pm 16.544$ & 0.123 & 0.1202 \\
\hline \multirow{2}{*}{$\begin{array}{c}\text { Wpbc } \\
(116 \times 33,78 \times 33)\end{array}$} & $\begin{array}{c}76.9643 \pm 22.3583 \\
\left(10^{\wedge}-5,2^{\wedge}-2\right)\end{array}$ & $\begin{array}{c}79.4643 \pm 16.544 \\
\left(10^{\wedge}-2,2^{\wedge}-1\right)\end{array}$ & $\begin{array}{c}\left(10^{\wedge}-2,2^{\wedge}-1\right) \\
0.0199\end{array}$ & $\begin{array}{c}8.2143 \pm 18.3426 \\
\left(10^{\wedge}-1,2^{\wedge}-1,0.1\right)\end{array}$ & $\begin{array}{c}81.9643 \pm 17.6466 \\
\left(10^{\wedge}-4,2^{\wedge}-1,0.1\right)\end{array}$ \\
\hline & 0.117 & 0.015 & & 0.0155 & 0.0135 \\
\hline
\end{tabular}

TABLE III. Average ranks of TWSVM, FTSVM, TPMSVM and FLTPMSVM using Gaussian kernel on real-world datasets

\begin{tabular}{|c|c|c|c|c|c|}
\hline $\begin{array}{c}\text { Dataset } \\
\text { (Train size, Test size) }\end{array}$ & SVM & TWSVM & FTSVM & TPMSVM & FLTPMSVM \\
\hline Australian-Credit & 5 & 4 & 1 & 3 & 2 \\
\hline Breast-Cancer BUPA & 4.5 & 4.5 & 3 & 2 & 1 \\
\hline liver Cleveland & 5 & 4 & 3 & 2 & 1 \\
\hline Haberman & 5 & 2 & 1 & 4 & 3 \\
\hline Heart-Statlog & 5 & 2 & 3 & 4 & 1 \\
\hline Ionosphare & 3 & 4.5 & 4.5 & 2 & 1 \\
\hline Pima-indians-diabetes & 5 & 2 & 2 & 4 & 2 \\
\hline Transfusion & 2 & 1 & 3 & 4 & 5 \\
\hline $\mathrm{Wpbc}$ & 1 & 3.5 & 3.5 & 5 & 2 \\
\hline \multirow[t]{2}{*}{ Average Rank } & 5 & 2.5 & 2.5 & 4 & 1 \\
\hline & 4.05 & 3 & 2.65 & 3.4 & 1.9 \\
\hline
\end{tabular}


FLTPMSVM is obtained by using simple linearly convergent iterative approach instead of solving two QPPs as in the case of TWSVM, FTSVM and TPMSVM. Hence, FLTPMSVM achieves a comparatively lower training cost as compared to the others. Further, the average ranks of all the methods are shown in Table 3, where rank is calculated on the basis of accuracies. One can observe from this table that proposed FLTPMSVM has the lowest average rank among all methods.

\section{CONCLUSION}

In this paper, a fuzzy-based Lagrangian twin parametricmargin support vector machine (FLTPMSVM) is proposed to lessen the effect of the outliers, by applying the concept of fuzzy support vector machine (FSVM) [15] on twin parametric-margin support vector machine (TPMSVM) [14]. Furthermore, the solution of FLTPMSVM is obtained by solving simple linearly convergent iterative schemes instead of solving a pair of QPPs as in case of TWSVM, FTSVM and TPMSVM. Experiments are carried out for non-linear case on publicly available real-world datasets as well as on one artificial dataset. Result shows that FLTPMSVM delivers comparative or better classification accuracy with the other considered methods and also suitable for heteroscedastic error structure. Moreover, our proposed method could achieve a faster training time as compared to all the other reported algorithms for all the datasets considered. Similar to TPMSVM, our proposed method loses the sparseness that can be one of the future works.

\section{REFERENCES}

[1]. V.N. Vapnik, 'Statistical Learning Theory," John Wiley \& Sons, New York, 1998.

[2]. T. Joachims, C. Ndellec \& C. Rouveriol, "Text categorization with support vector machines: learning with many relevant features," in European Conference on Machine Learning No.10, Chemnitz, Germany, pp.137$142,1998$.

[3]. C. Cortes \& V. Vapnik, "Support-Vector Networks, Machine Learning," 20(6), pp.273-297, 1995

[4]. R. Khemchandani \& S. Sharma, "Robust Least Squares Twin Support Vector Machine for Human Activity Recognition," Applied Soft Computing, 47, pp.33-46, 2016.

[5]. Y-K. Bao, Z-T. Liu, L. Guo \& W. Wang, "Forecasting stock composite index by fuzzy support vector machines regression," in Proceeding of International Conference on Machine Learning and Cybernetics, 6, pp. $3535-3540,2005$.

[6]. T. Ebrahimi, G. N. Garcia \& J.M. Vesin, "Joint time-frequency-space classification of EEG in a brain-computer interface application," Journal of Applied Signal Processing, vol. 1(10), pp.713-729, 2003.

[7]. R. Malhotra, \& D.K. Malhotra, "Evaluating consumer loans using neural networks,” Omega 31, pp.83-96, 2003.

[8]. Jayadeva, R. Khemchandani, \& S. Chandra, "Twin support vector machines for pattern classification," IEEE Transactions on Pattern Analysis and Machine Intelligence, vol. 29(8), pp.905-910, 2007.

[9]. J.A.K. Suykens \& J. Vandewalle, "Least squares support vector machine classifiers," Neural Processing Letters, 9(6), pp.293-300, 1999.

[10]. Mangasarian, Olvi L., and David R. Musicant. "Lagrangian support vector machines." Journal of Machine Learning Research 1.Mar (2001): 161-177.

[11]. Balasundaram, S., Deepak Gupta, and Subhash Chandra Prasad. "A new approach for training Lagrangian twin support vector machine via unconstrained convex minimization." Applied Intelligence 46.1 (2017): 124-134.

[12]. P-Y. Hao, "New support vector algorithms with parametric insensitive/margin model," Neural Networks, 23 (4), pp.60-73, 2010.
[13]. B. Scholkopf, A.J. Smola, R.C. Williamson \& P.L. Bartlett, "New Support Vector Algorithms," Neural Computation, 12 (8), pp.1207- 1245, 2000.

[14]. Peng, X., (2011), "TPMSVM: A novel twin parametric-margin support vector machine for pattern recognition," Pattern Recognition, 44 pp.2678-2692.

[15]. C.-F. Lin \&, S.-D. Wang, "Fuzzy Support Vector Machines," IEEE Trans. Neural Networks, vol. 13(5), pp. 464-471 (2002).

[16]. R. Batuwita \& V. Palade, "FSVM-CIL: Fuzzy Support Vector Machines for Class Imbalance Learning," IEEE Trans. Fuzzy Systems, vol. 18, no. 3, pp. 558-571, June (2010).

[17]. D. Tsujinishi \& S. Abe, "Fuzzy least squares support vector machines," Proceedings of the International Joint Conference on Neural Networks, Portland, Oregon, pp. 1599-1604, (2003).

[18]. Y. Wang, S. Wang \& K. K. Lai, "A new fuzzy support vector machine to evaluate credit risk." IEEE Transactions on Fuzzy Systems, Vol. 13(9), pp. 820-831 (2005).

[19]. Chaudhuri \& K. De, "Fuzzy support vector machine for bankruptcy prediction," Applied Soft Computing, 11(5), pp. 2472-2486 (2010).

[20]. S. Zhang, S. Zhao, Y. Sui \& L. Zhang, "Single object tracking with fuzzy least squares support vector machine." IEEE Transactions Image Processing, Vol. 24, pp. 5723-5738 (2015)

[21]. N. Cristianini \& J. Shawe-Taylor, "An introduction to support vector machines and other kernel based learning methods," Cambridge University Press, Cambridge, 2000.

[22]. R. Batuwita \& V. Palade, "FSVM-CIL: Fuzzy Support Vector Machines for Class Imbalance Learning", IEEE Trans. Fuzzy Systems, vol. 18, no. 3, pp. 558-571, June (2010).

[23]. O.L. Mangasarian, "Nonlinear Programming," SIAM Philadelphia, PA, 1994.

[24]. O.L. Mangasarian, \& D.R. Musicant, "Lagrangian support vector machines,' Journal of Machine Learning Research, 1, pp.161-177, 2001.

[25]. B.D. Ripley, "pattern recognition and Neural Networks," Cambridge university Press, Cambridge, 1996.

[26]. P.M. Murphy \& D.W. Aha, "UCI repository of machine learning databases," University of California, Irvine. 1992, http://www.ics.uci.edu/ mlearn. 